ANNALES

POLONICI MATHEMATICI

$83.1(2004)$

\title{
Mean lower bounds for Markov operators
}

\author{
by EduARd Emel'yanov (Novosibirsk and Tübingen) \\ and Manfred WolfF (Tübingen)
}

\begin{abstract}
Let $T$ be a Markov operator on an $L^{1}$-space. We study conditions under which $T$ is mean ergodic and satisfies $\operatorname{dim} \operatorname{Fix}(T)<\infty$. Among other things we prove that the sequence $\left(n^{-1} \sum_{k=0}^{n-1} T^{k}\right)_{n}$ converges strongly to a rank-one projection if and only if there exists a function $0 \neq h \in L_{+}^{1}$ which satisfies $\lim _{n \rightarrow \infty}\left\|\left(h-n^{-1} \sum_{k=0}^{n-1} T^{k} f\right)_{+}\right\|=0$ for every density $f$. Analogous results for strongly continuous semigroups are given.
\end{abstract}

Preliminaries. Let $(\Omega, \Sigma, \mu)$ be a $\sigma$-finite measure space, and $L^{1}=$ $L^{1}(\Omega, \Sigma, \mu)$ the space of all real-valued Lebesgue-integrable functions on $(\Omega, \Sigma, \mu)$. In the following we will use the notation $\mathbb{1}_{A}$ for the characteristic function of the set $A \in \Sigma$. By $\mathcal{D}=\mathcal{D}(\Omega, \Sigma, \mu)$ we will denote the set of all densities on $\Omega$, that is,

$$
\mathcal{D}=\left\{f \in L_{+}^{1}:\|f\|=1\right\} .
$$

A linear operator $T: L^{1} \rightarrow L^{1}$ is called a Markov operator if $T(\mathcal{D}) \subseteq \mathcal{D}$. The study of the asymptotic properties of Markov chains leads to questions of convergence of $\left(\mathcal{A}_{n}(T) f\right)_{n}$ for $f \in L^{1}$, where

$$
\mathcal{A}_{n}(T):=\frac{1}{n} \sum_{k=0}^{n-1} T^{k}
$$

denotes the Cesàro means of $T$. A Markov operator $T$ is called mean ergodic if the norm limit $\lim _{n \rightarrow \infty} \mathcal{A}_{n}(T) f$ exists for all $f \in L^{1}$.

1. Main results. In many cases it is important to find conditions under which a Markov operator $T$ is mean ergodic and the space Fix $(T)$ of all $T$ fixed vectors has finite dimension. For this the following theorem is useful.

ThEOREM 1. Let $T$ be a Markov operator on $L^{1}(\Omega, \Sigma, \mu)$. Then the following assertions are equivalent:

2000 Mathematics Subject Classification: 37A30, 47A35.

Key words and phrases: Markov operator, mean ergodicity, mean lower-bound function. 
(i) There exists an element $y \in L_{+}^{1}$ and a real number $\eta$ with $0<\eta<1$ such that

$$
\varlimsup_{n \rightarrow \infty}\left\|\left(\mathcal{A}_{n}(T) f-y\right)_{+}\right\| \leq \eta
$$

for every density $f$.

(ii) There is an integer $m$, a decomposition $\Omega=\bigcup_{k=0}^{m} \Omega_{k}$ into pairwise disjoint subsets $\Omega_{k} \in \Sigma$, and two sequences of nonnegative functions $u_{k}=$ $T u_{k} \in \mathcal{D}\left(\Omega_{k}\right)$ and $\phi_{k} \in L^{\infty}\left(\Omega_{0}\right), k=1, \ldots, m$, such that $\sum_{k=1}^{m} \phi_{k}=\mathbb{1}_{\Omega_{0}}$ and for every $g \in L^{1}(\Omega)$ the norm limit $\lim _{n \rightarrow \infty} \mathcal{A}_{n}(T) g$ exists and it may be written in the form

$$
\lim _{n \rightarrow \infty} \mathcal{A}_{n}(T) g=\sum_{k=1}^{m}\left[\int_{\Omega}\left(\phi_{k}+\mathbb{1}_{\Omega_{k}}\right) g d \mu\right] u_{k}
$$

moreover, $\lim _{n \rightarrow \infty} \int_{\Omega_{0}} T^{n} g d \mu=0$.

Proof. (i) $\Rightarrow$ (ii). Set $E:=L^{1}(\Omega, \Sigma, \mu)$. We show that $T$ is mean ergodic. By Sine's ergodic theorem (see $[\mathrm{Kr}, \mathrm{p} .74]$ ) it is enough to check that for every $T^{*}$-fixed point $0 \neq \psi \in E^{*}=L^{\infty}(\Omega, \Sigma, \mu)$ there exists a $T$-fixed point $w \in E$ which satisfies $\langle\psi, w\rangle \neq 0$.

Let $E^{*} \ni \psi \neq 0, T^{*} \psi=\psi$. We may assume that $\left\|\psi_{+}\right\|=\|\psi\|=1$. Set $\varepsilon:=(1-\eta) / 3$ and take some $f \in E$ which satisfies $\|f\|=1$ and $\left\langle\psi_{+}, f\right\rangle \geq 1-\varepsilon$. We have \|\|$f\|\|=\|f\|=1$ and

$$
1 \geq\langle|\psi|,|f|\rangle \geq\left\langle\psi_{+},|f|\right\rangle \geq\left\langle\psi_{+}, f\right\rangle \geq 1-\varepsilon
$$

Consequently,

$$
\langle\psi,|f|\rangle=\left\langle 2 \psi_{+},|f|\right\rangle-\langle|\psi|,|f|\rangle \geq 2(1-\varepsilon)-1=1-2 \varepsilon .
$$

Let $f^{\prime \prime} \in E^{* *}$ be a $w^{*}$-cluster point of $\left(\mathcal{A}_{n}(T)|f|\right)_{n=1}^{\infty}$. Then $f^{\prime \prime}$ obviously satisfies $T^{* *} f^{\prime \prime}=f^{\prime \prime}$. Since

$$
\varlimsup_{n \rightarrow \infty} \operatorname{dist}\left(\mathcal{A}_{n}(T)|f|,[0, y]\right) \leq \eta
$$

and $[0, y]$ is weakly compact in $E$, we obtain

$$
f^{\prime \prime} \in[0, y]+\eta B_{E^{* *}} \subseteq E+\eta B_{E^{* *}},
$$

where as usual $B_{E^{* *}}$ denotes the unit ball of $E^{* *}$. Take the canonical projection $P: E^{* *} \rightarrow E$. Then $(I-P) f^{\prime \prime} \in \eta B_{E^{* *}}$, and

$$
\begin{aligned}
\left\langle\psi, P f^{\prime \prime}\right\rangle & =\left\langle\psi_{+}, P f^{\prime \prime}\right\rangle-\left\langle\psi_{-}, P f^{\prime \prime}\right\rangle \\
& =\left\langle f^{\prime \prime}, \psi_{+}\right\rangle-\left\langle(I-P) f^{\prime \prime}, \psi_{+}\right\rangle-\left\langle\psi_{-}, P f^{\prime \prime}\right\rangle \\
& \geq\left\langle f^{\prime \prime}, \psi\right\rangle-\eta=\langle\psi,|f|\rangle-\eta \geq 1-2 \varepsilon-\eta=\varepsilon>0 .
\end{aligned}
$$

Moreover

$$
T P f^{\prime \prime}=T P\left(T^{m}\right)^{* *} f^{\prime \prime} \geq T P\left(T^{m}\right)^{* *} P f^{\prime \prime}=T P T^{m} P f^{\prime \prime}=T^{m+1} P f^{\prime \prime} \geq 0 .
$$


Thus the sequence $\left(T^{m} P f^{\prime \prime}\right)_{m}$ is decreasing in $E=L^{1}(\Omega, \Sigma, \mu)$, and hence $w:=\lim _{m \rightarrow \infty} T^{m} P f^{\prime \prime}$ exists. Clearly $T w=w$, and $\langle\psi, w\rangle=\left\langle\psi, P f^{\prime \prime}\right\rangle>0$. Thus $T$ is mean ergodic.

The space $\operatorname{Fix}(T)$ is an $L^{1}$-space as the range of a Markov projection. Since

$$
\left\|(z-y)_{+}\right\| \leq \eta<1 \quad(\forall z \in \mathcal{D} \cap \operatorname{Fix}(T))
$$

which implies

$$
\|y\| \geq\|z \wedge y\| \geq 1-\eta>0 \quad(\forall z \in \mathcal{D} \cap \operatorname{Fix}(T)),
$$

we obtain $\operatorname{dim} \operatorname{Fix}(T)<\infty$. Take a maximal pairwise disjoint family $\left\{u_{k}\right\}_{k=1}^{m}$ of $T$-invariant densities (it is clear that $m=\operatorname{dim} \operatorname{Fix}(T)$ ), and put

$$
\Omega_{k}:=\left\{x \in \Omega: u_{k}(x)>0\right\}, \quad \Omega_{0}:=\Omega-\bigcup_{k=0}^{m} \Omega_{k} .
$$

Let $Q$ be the strong limit of $\left(\mathcal{A}_{n}(T)\right)_{n}$. Then $Q$ may be written in the form

$$
Q g=\sum_{k=1}^{m} \lambda_{k}(g) u_{k} \quad\left(\forall g \in L^{1}\right),
$$

where $\lambda_{k}$ are positive linear functionals on $L^{1}$, which we may consider as elements of $L^{\infty}$. Since $Q u_{k}=u_{k}$ we obtain

$$
u_{k}=\left[\int_{\Omega} \lambda_{k} \cdot u_{k} d \mu\right] u_{k} .
$$

Hence $\lambda_{k} \cdot \mathbb{1}_{\Omega_{k}}=\mathbb{1}_{\Omega_{k}}$. Put $\phi_{k}=\lambda_{k}-\mathbb{1}_{\Omega_{k}}$. Then assertion (ii) is true for $m$, $\Omega_{k}, u_{k}$, and $\phi_{k}$.

Given $g \in L_{+}^{1}$, the sequence $\left(\left\|\mathbb{1}_{\Omega_{0}} \cdot T^{n} g\right\|\right)_{n}$ is obviously decreasing, and satisfies $\lim _{n \rightarrow \infty}\left\|\mathbb{1}_{\Omega_{0}} \cdot \mathcal{A}_{n}(T) g\right\|=0$. Hence the inequality

$$
\inf _{n}\left\|\mathbb{1}_{\Omega_{0}} \cdot T^{n} g\right\| \leq \frac{1}{n} \sum_{k=0}^{n-1}\left\|\mathbb{1}_{\Omega_{0}} \cdot T^{k} g\right\|=\left\|\mathbb{1}_{\Omega_{0}} \cdot \mathcal{A}_{n}(T) g\right\|
$$

implies that $\lim _{n \rightarrow \infty} \int_{\Omega_{0}} T^{n} g d \mu=0$.

(ii) $\Rightarrow$ (i). This is an easy exercise.

We point out that our proof shows that in fact the assertion of the theorem is also true for arbitrary positive operators on $L^{1}(X, \Sigma, \mu)$.

In some applications of Theorem 1 it seems to be easier to check an integral form of (1). Originally such a condition was introduced and exploited by Komornik, Lasota, and Socała [Ko], [KL], [LS]. A stronger but similar integral condition was used by Bartoszek [Ba1].

THEOREM 2. Under the same conditions as in Theorem 1 assertions (i) and (ii) are equivalent to: 
(iii) There exist $\delta>0$ and $\eta<1$ and a set $A \in \Sigma$ with $\mu(A)<\infty$ such that for every density $f$ there is an integer $n(f)$ for which

$$
\int_{(\Omega-A) \cup B} \mathcal{A}_{n}(T) f d \mu \leq \eta
$$

for $n \geq n(f)$ and $\mu(B) \leq \delta$.

Proof. $\left(\right.$ iii) $\Rightarrow(\mathrm{i})$. Let $\mathcal{X}:=\left\{\mathcal{A}_{n}(T) f: f \in L_{+}^{1},\|f\|=1, n \geq n(f)\right\}$. Obviously $\mathcal{X}$ is norm bounded in $L^{1}$ by 1 . Take some $A \in \Sigma$ of finite measure, $\eta<1$ and $\delta>0$ which satisfy $\int_{B \cup(\Omega-A)} g d \mu \leq \eta$ for all $g \in \mathcal{X}$ whenever $\mu(B) \leq \delta$. Suppose that (i) does not hold. Then for each $k \in \mathbb{N}$ there is an element $g_{k} \in \mathcal{X}$ such that $\left\|\left(g_{k}-k \mathbb{1}_{A}\right)_{+}\right\|>1-1 / k$. Hence

$$
\begin{aligned}
1 & \leq \varlimsup_{k \rightarrow \infty}\left\|\left(g_{k}-k \mathbb{1}_{A}\right)_{+}\right\|=\varlimsup_{k \rightarrow \infty}\left[\int_{\Omega-A} g_{k} d \mu+\int_{A}\left(g_{k}-k \mathbb{1}_{A}\right)_{+} d \mu\right] \\
& \leq \varlimsup_{k \rightarrow \infty}\left[\int_{\Omega-A} g_{k} d \mu+\int_{B_{k}}:=\left\{x \in \Omega: g_{k}(x) \geq k\right\}\right. \\
& =\varlimsup_{k \rightarrow \infty} \int_{B_{k} \cup(\Omega-A)} g_{k} d \mu \leq \eta<1,
\end{aligned}
$$

since $\mu\left(B_{k}\right) \leq 1 / k \leq \delta$ for large $k$. The contradiction shows that (i) holds.

(i) $\Rightarrow$ (iii). Let (i) be satisfied by an element $h \in L_{+}^{1}$ and $\eta<1$. Set $\mathcal{Y}:=[-h, h]+\eta B_{L^{1}}$, where $B_{L^{1}}$ is the unit ball of $L^{1}$. Then $\left\|(|f|-h)_{+}\right\| \leq \eta$ for all $f \in \mathcal{Y}$. Take a set $A \in \Sigma$ of finite measure and $\delta>0$ which satisfy $\int_{B \cup(\Omega-A)} y d \mu \leq(1-\eta) / 4$ for all $B \in \Sigma$ with $\mu(B)<\delta$. Then we have

$$
\begin{aligned}
\int_{B \cup(\Omega-A)} \mathcal{A}_{n}(T) f d \mu & \leq \int_{\Omega}\left(\mathcal{A}_{n}(T) f-h\right)_{+} d \mu+\int_{B \cup(\Omega-A)} h d \mu \\
& \leq\left\|\left(\mathcal{A}_{n}(T) f-h\right)_{+}\right\|+(1-\eta) / 4 \\
& <\eta+(1-\eta) / 2=(1+\eta) / 2<1
\end{aligned}
$$

for all $f \in X_{+}$with $\|f\|=1$ whenever $\mu(B)<\delta$ and $n \geq n(f)$ is large enough.

To complete the picture we give a strongly continuous variant of Theorems 1 and 2 .

TheOREM 3. Let $\mathcal{T}=\left(T_{t}\right)_{t \geq 0}$ be a strongly continuous Markov semigroup on $L^{1}(\Omega, \Sigma, \mu)$. Then the following assertions are equivalent:

(i) There exist $h \in L_{+}^{1}$ and $\eta<1$ such that

$$
\varlimsup_{t \rightarrow \infty}\left\|\left(\frac{1}{t} \int_{0}^{t} T_{\tau} f d \tau-h\right)_{+}\right\| \leq \eta
$$

for every density $f$. 
(ii) There is an integer $m$, a decomposition $\Omega=\bigcup_{k=0}^{m} \Omega_{k}$ into pairwise disjoint subsets $\Omega_{k} \in \Sigma$, and two sequences of nonnegative functions $u_{k}=$ $\mathcal{T} u_{k} \in \mathcal{D}\left(\Omega_{k}\right)$ and $\phi_{k} \in L^{\infty}\left(\Omega_{0}\right), k=1, \ldots, m$, such that $\sum_{k=1}^{m} \phi_{k}=\mathbb{1}_{\Omega_{0}}$ and for every $g \in L^{1}(\Omega)$ the norm limit $\lim _{t \rightarrow \infty} \frac{1}{t} \int_{0}^{t} T_{\tau} g d \tau$ exists and it may be written in the form

$$
\lim _{t \rightarrow \infty} \frac{1}{t} \int_{0}^{t} T_{\tau} g d \tau=\sum_{k=1}^{m}\left[\int_{\Omega}\left(\phi_{k}+\mathbb{1}_{\Omega_{k}}\right) g d \mu\right] u_{k}
$$

moreover, $\lim _{t \rightarrow \infty} \int_{\Omega_{0}} T_{t} g d \mu=0$.

(iii) There exist $\delta>0$ and $\eta<1$ and a set $A \in \Sigma$ with $\mu(A)<\infty$ such that for every density $f$ there is $r(f) \geq 0$ for which

$$
\int_{(\Omega-A) \cup B}\left[\frac{1}{r} \int_{0}^{r} T_{\tau} f d \tau\right] d \mu \leq \eta
$$

for $r \geq r(f)$ and $\mu(B) \leq \delta$.

The proof of Theorem 3 is similar to the proofs of Theorems 1 and 2 and we drop it. Notice also that Theorem 3 can be easily reduced to Theorems 1,2 .

Let us point out that in all theorems of this section it is enough to consider only a dense subset of $\mathcal{D}$ instead of $\mathcal{D}$.

2. Applications. In order to show the power of Theorem 1 we give two corollaries of it, the first of them being well known as Lasota's lower bound criteria [La] of asymptotic stability. The other one is new.

Following Lasota we call a Markov operator $T$ statistically stable whenever there exists a density $g$ such that

$$
\lim _{n \rightarrow \infty}\left\|T^{n} f-g\right\|=0 \quad(\forall f \in \mathcal{D}),
$$

and we call a function $h \in L_{+}^{1}$ a lower-bound function for $T$ if

$$
\lim _{n \rightarrow \infty}\left\|\left(h-T^{n} f\right)_{+}\right\|=0 \quad(\forall f \in \mathcal{D}) .
$$

Finally, we say that $h$ is nontrivial if $h \neq 0$. The following result is due to Lasota [La, Theorem 1.1].

TheOREM 4 (Lasota). Let $T$ be a Markov operator on $L^{1}$. Then the following assertions are equivalent:

(i) $T$ is statistically stable.

(ii) There exists a nontrivial lower-bound function for $T$.

Proof. (i) $\Rightarrow$ (ii). Let a density $h \in L^{1}$ satisfy

$$
\lim _{n \rightarrow \infty}\left\|T^{n} f-h\right\|=0 \quad(\forall f \in \mathcal{D}) .
$$

Then $h$ is a nontrivial lower-bound function for $T$. 
(ii) $\Rightarrow$ (i). Let $0 \neq h \in L_{+}^{1}$ be a nontrivial lower-bound function for $T$. Set as usual

$$
L_{0}^{1}:=\left\{f \in L^{1}:\left\|f_{+}\right\|=\left\|f_{-}\right\|\right\} .
$$

Since $h$ is a nontrivial lower-bound function, we obtain easily

$$
\varlimsup_{n \rightarrow \infty}\left\|\left(\mathcal{A}_{n}(T) f-h\right)_{+}\right\| \leq 1-\|h\|<1 \quad(\forall f \in \mathcal{D}),
$$

and hence $T$ is mean ergodic due to Theorem 1 . Then there exists a $T$ invariant density, say $g$. Since $L^{1}=L_{0}^{1} \oplus \mathbb{R} \cdot g$, it is enough to show that

$$
\lim _{n \rightarrow \infty}\left\|T^{n} f\right\|=0 \quad\left(\forall f \in L_{0}^{1}\right) .
$$

Notice that $\left(\left\|T^{n} f\right\|\right)_{n}$ is a monotone sequence since $T$ is a contraction. Hence $\|f\| \geq \lim _{n \rightarrow \infty}\left\|T^{n} f\right\|=\inf _{n}\left\|T^{n} f\right\|$ for every $f$. Now suppose that there exists an $f \in L_{0}^{1}$ with $2 \alpha:=\lim _{n \rightarrow \infty}\left\|T^{n} f\right\|>0$. Then

$$
\begin{aligned}
2 \alpha & =\lim _{n \rightarrow \infty}\left\|T^{n} f\right\|=\lim _{n \rightarrow \infty}\left\|T^{n}\left(f_{+}-f_{-}\right)\right\| \\
& =\lim _{n \rightarrow \infty}\left\|\left(T^{n} f_{+}-\alpha h\right)_{+}-\left(T^{n} f_{-}-\alpha h\right)_{+}\right\| \\
& \leq \lim _{n \rightarrow \infty}\left(\left\|\left(T^{n} f_{+}-\alpha h\right)_{+}\right\|+\left\|\left(T^{n} f_{-}-\alpha h\right)_{+}\right\|\right) \\
& =2 \alpha(1-\|h\|),
\end{aligned}
$$

which is impossible (notice that (8) is true because $h$ is a lower-bound function and $\left\|f_{+}\right\|=\left\|f_{-}\right\| \geq \alpha$ ). Consequently, the condition (7) holds.

An analogous result also holds for continuous-time parametrized semigroups. For an elegant proof we refer to [LM, Thm. 7.4.1].

We call an $h \in L_{+}^{1}$ a mean lower-bound function for a Markov operator $T$ if

$$
\lim _{n \rightarrow \infty}\left\|\left(h-\mathcal{A}_{n}(T) f\right)_{+}\right\|=0 \quad(\forall f \in \mathcal{D}) .
$$

Obviously any lower-bound function is mean lower-bound. A special kind of nontrivial mean lower bounds was used by W. Bartoszek [Ba2]. His additional assumptions on the operator $T$ imply that it is asymptotically periodic.

TheOREM 5. Let $T$ be a Markov operator on $L^{1}$. Then the following assertions are equivalent:

(i) There exists a density $g$ such that

$$
\lim _{n \rightarrow \infty}\left\|\mathcal{A}_{n}(T) f-g\right\|=0 \quad(\forall f \in \mathcal{D}) .
$$

(ii) There exists a nontrivial mean lower-bound function for $T$.

Proof. (i) $\Rightarrow$ (ii). Let $g$ satisfy $\lim _{n \rightarrow \infty}\left\|\mathcal{A}_{n}(T) f-g\right\|=0$ for all $f \in \mathcal{D}$. Then $g$ is a nontrivial mean lower-bound function for $T$. 
(ii) $\Rightarrow$ (i). Let $0 \neq h \in L_{+}^{1}$ be a nontrivial mean lower-bound function for $T$. Then

$$
\varlimsup_{n \rightarrow \infty}\left\|\left(\mathcal{A}_{n}(T) f-h\right)_{+}\right\| \leq \eta \quad(\forall f \in \mathcal{D})
$$

with $\eta:=1-\|h\|$. By Theorem $1, T$ is mean ergodic, so we obtain a decomposition

$$
L^{1}=\operatorname{Fix}(T) \oplus \overline{(I-T) L^{1}} .
$$

All that we need is $\operatorname{dim}(\operatorname{Fix}(T))=1$. By Theorem $1, \operatorname{Fix}(T)$ is spanned by $\operatorname{dim}(\operatorname{Fix}(T))=p$ densities $u_{k}$, which in case $p>1$ satisfy

$$
\inf \left(u_{i}, u_{k}\right)=0 \quad \text { for } i \neq k \text {. }
$$

But $T u_{k}=u_{k}$ implies $\left\|\left(h-u_{k}\right)_{+}\right\|=\lim _{n \rightarrow \infty}\left\|\left(h-\mathcal{A}_{n}(T) u_{k}\right)_{+}\right\|=0$, hence $u_{k} \geq h>0$ for all $k \leq p$, which by (11) is only possible in case $p=1$.

The strongly continuous variant of Theorem 5 may be obtained similarly by using Theorem 3, or it can be derived from Theorem 5 . It reads as follows.

Theorem 6. Let $\mathcal{T}=\left(T_{t}\right)_{t \geq 0}$ be a strongly continuous Markov semigroup on $L^{1}$. Then the following assertions are equivalent:

(i) There exists a density $g$ such that

$$
\lim _{t \rightarrow \infty}\left\|\frac{1}{t} \int_{0}^{t} T_{\tau} f d \tau-g\right\|=0 \quad(\forall f \in \mathcal{D}) .
$$

(ii) There exists an $0 \neq h \in L_{+}^{1}$ that satisfies

$$
\lim _{t \rightarrow \infty}\left\|\left(h-\frac{1}{t} \int_{0}^{t} T_{\tau} f d \tau\right)_{+}\right\|=0 \quad(\forall f \in \mathcal{D}) .
$$

To complete the picture we give two different applications of Theorem 5 . As usual, let $(\Omega, \Sigma, \mu)$ be a $\sigma$-finite measure space and let $K: \Omega \times \Omega \rightarrow \mathbb{R}_{+}$ be a Markov kernel. Define $T: L^{1}(\Omega, \Sigma, \mu) \rightarrow L^{1}(\Omega, \Sigma, \mu)$ by

$$
T f(x)=\int K(x, y) f(y) d y
$$

and by induction

$$
K_{1}(x, y)=K(x, y), \quad K_{n+1}(x, y)=\int K(x, z) K_{n}(z, y) d z .
$$

Then $T^{n} f(x)=\int K_{n}(x, y) f(y) d y$. Finally, we set

Then

$$
\bar{K}_{n}(x, y)=\frac{1}{n} \sum_{k=1}^{n} K_{k}(x, y) \text {. }
$$

$$
T \mathcal{A}_{n}(T) f(x)=: A_{n} f(x)=\frac{1}{n} \sum_{k=1}^{n} T^{k} f(x)=\int \bar{K}_{n}(x, y) f(y) d y .
$$

We obtain the following proposition. 
Proposition 7. Assume that $\int \liminf _{n \rightarrow \infty}\left(\inf _{y} \bar{K}_{n}(x, y)\right) d x>0$. Then $T$ is mean ergodic and the space of its fixed vectors is one-dimensional.

Proof. Set $h(x)=\liminf _{n \rightarrow \infty} \inf _{y} \bar{K}_{n}(x, y)$. Then $h \neq 0$ by hypothesis. Moreover an easy computation shows that $h$ is a mean lower bound. Now apply Theorem 5 .

Let $(\Omega, \Sigma, \mu)$ as well as $K(x, y), T$, and $\bar{K}_{n}(x, y)$ be as before. Let $V$ : $\Omega \rightarrow \mathbb{R}_{+}$be an arbitrary measurable function which is not a null function with respect to $\mu$, and set $G_{a}:=V^{-1}([0, a])=\{x \in \Omega: V(x) \leq a\}$.

Proposition 8. Assume that there exists a constant $M>0$ and a subset $\mathcal{D}_{0}$ of the set $\mathcal{D}$ of all densities which is dense in $\mathcal{D}$ such that

$$
\limsup _{n \rightarrow \infty} \iint V(x) \bar{K}_{n}(x, y) f(y) d y d x \leq M \quad \text { for all } f \in \mathcal{D}_{0} .
$$

Moreover assume that for every $a>0$ with $G_{a} \neq \emptyset$,

$$
\int_{y \in G_{a}} K(x, y) d x=\delta(a)>0 .
$$

Then $T$ is mean ergodic and the space of its fixed vectors is one-dimensional.

Proof. Let $f$ be in $\mathcal{D}_{0}$. Then there exists $n_{0}$ such that

$$
\iint V(x) \bar{K}_{n}(x, y) f(y) d y d x \leq M+1
$$

for all $n \geq n_{0}$. Choose $a \geq 3 M$ such that $G_{a} \neq \emptyset$. Then by Chebyshev's inequality (cf. [LM, Prop. 5.7.1]) we obtain, for $A_{n}:=n^{-1} \sum_{k=1}^{n} T^{k}=$ $T \mathcal{A}_{n}(T)$,

$$
\int_{G_{a}} A_{n} f d x \geq 1-\frac{1}{a} \int V A_{n} f d x>1-\frac{M+1}{3 M}>1 / 2
$$

for $n \geq n_{0}$. Moreover $A_{n+1} \geq \frac{n}{n+1} T A_{n}$. Hence

$$
A_{n+1} f(x) \geq \frac{n}{n+1} \int K(x, y) A_{n} f(y) d y \geq \frac{n}{n+1} \int_{G_{a}} K(x, y) A_{n} f(y) d y .
$$

Therefore for $h(x)=\inf _{y \in G_{a}} K(x, y)$ we obtain

$$
A_{n+1} f(x) \geq \frac{n}{n+1} \inf _{y \in G_{a}} K(x, y) \int_{G_{a}} A_{n} f(y) d y \geq \frac{n}{n+1} \cdot \frac{1}{2} \cdot h(x) \geq \frac{h(x)}{4} .
$$

Thus $\lim _{n \rightarrow \infty}\left\|\left(A_{n} f-h / 4\right)_{-}\right\|=0$ for all $f \in \mathcal{D}_{0}$. But since $\mathcal{D}_{0}$ is dense in $\mathcal{D}$, and $h \neq 0$ by hypothesis, $h / 4$ is a mean lower bound and the assertion follows from Theorem 5 .

REMARK. If $V$ is bounded then the hypotheses on $T$ imply that $T$ dominates an operator $1_{\Omega} \otimes h$, from which it follows easily that $h$ is a lower 
bound, and hence $T$ is not only ergodic but even statistically stable by Theorem 4. So Proposition 8 is only interesting in the case of $V$ unbounded (cf. also [LM, Theorem 5.7.2]).

Acknowledgements. The first author is grateful for generous support of the Alexander von Humboldt Foundation during his stay at the University of Tübingen. Both authors are grateful for the remarks of the referee which helped to improve the paper.

\section{References}

[Ba1] W. Bartoszek, On uniformly smoothing stochastic operators, Comment. Math. Univ. Carolin. 36 (1995), 203-206.

[Ba2] - Convergence of iterates of Lasota-Mackey-Tyrcha operators, Ann. Polon. Math. 63 (1996), 281-292.

[Ko] J. Komornik, Asymptotic periodicity of the iterates of weakly constrictive Markov operators, Tohoku Math. J. (2) 38 (1986), 15-27.

[KL] J. Komornik and A. Lasota, Asymptotic decomposition of Markov operators, Bull. Polish Acad. Sci. Math. 35 (1987), 321-327.

[Kr] U. Krengel, Ergodic Theorems, de Gruyter, Berlin 1985.

[La] A. Lasota, Statistical stability of deterministic systems, in: Equadiff 82 (Würzburg, 1982), Lecture Notes in Math. 1017, Springer, Berlin, 1983, 386-419.

[LM] A. Lasota and M. C. Mackey, Chaos, Fractals, and Noise. Stochastic Aspects of Dynamics, 2nd ed., Appl. Math. Sci. 97, Springer, New York, 1994.

[LS] A. Lasota and J. Socała, Asymptotic properties of constrictive Markov operators, Bull. Polish Acad. Sci. Math. 35 (1987), 71-76.

Sobolev Institute of Mathematics

Mathematisches Institut

Akad. Koptyug pr. 4

630090 Novosibirsk, Russia

Universität Tübingen

E-mail: emelanov@math.nsc.ru

Auf der Morgenstelle 10

D-72076 Tübingen, Germany

E-mail: manfred.wolff@uni-tuebingen.de 